\title{
APONTAMENTOS TEÓRICOS E PRÁTICOS SOBRE A ANÁLISE DE MULTIMODALIDADE EM JOGOS DIGITAIS LOCALIZADOS
}

\author{
क्षिक \\ FERNANDO DA SILVA
}

Resumo: Este artigo apresenta alguns breves apontamentos teóricos e metodológicos acerca do mapeamento e análise de conteúdos multimodais em jogos de videogame localizados em pesquisas na área dos Estudos da Tradução e Estudos baseados em Corpora. A proposta de observação de conteúdos multimodais (áudio, vídeo, texto, imagens estáticas, etc.) em videogames localizados favorece um olhar ampliado sobre os diferentes aspectos de representação linguística, visual e cultural que emergem nesse objeto de estudos. Na formalização desta proposta faço uma breve introdução acerca de alguns recursos - como software de anotação de corpora multimodais, hardware de captura de vídeo entre outras ferramentas para investigação de corpora com características multimodais. Por fim, exponho as principais limitações associadas ao encaminhamento de investigações de corpora de natureza dinâmica como jogos digitais, e de que maneira a tarefa de observação da multiplicidade de recursos semióticos pode trazer à tona intertextualidade além do corpus analisado.

Palavras-chave: Análise de corpus. Multimodalidade. Localização de jogos.

\begin{abstract}
This article proposes to present brief theoretical and methodological contributions regarding the mapping and analysis of multimodal contents in localized videogames within the framework of Translation Studies and Corpus-based Studies. The proposal to observe multimodal contents (audio, video, texts, static images, etc) in localized videogames favors a deeper look into the different aspects of linguistic, visual and cultural representations that emerge in this type of investigation. In order to formalize the article's proposal I make a brief introduction on resources such as software for multimodal corpus annotation and hardware for video capture, amongst other tools, to be used for the investigation of multimodal corpora. Finally, I show the main limitations in investigating corpora of a dynamic nature, such as digital games, and in what ways the task of observing the multiplicity of semiotic resources brings to the fore intertextual relations beyond the analyzed corpus.
\end{abstract}

Keywords: Corpus analysis. Multimodality. Game localization. 
$\mathrm{P}$ ara vários autores, dentre os quais muitos dos citados neste texto, é perceptível uma deficiência nos aparatos metodológicos de análise de corpora multimodais que estabeleçam de maneira satisfatória uma interface com os Estudos da Tradução e estudos de multimodalidade que não apenas em nível de transcrição de diálogos, legendas e descrição de cenas. De forma semelhante à construção de um corpus de textos escritos, o processo de design e investigação de corpora multimodais implica na organização de vários aspectos de ordem conceitual e metodológica, que englobam, por exemplo, a seleção dos corpora para análise, a escolha de ferramentas, a delimitação dos aspectos a serem anotados, a anotação, a obtenção de dados, a análise, a interpretação e a discussão de resultados. Para O’Halloran et al (2012), para a interpretação de padrões semióticos emergentes - de texto, áudio, e vídeo - é necessário um aparato de recursos teóricopráticos uma vez que esse tipo de observação se dá a partir de uma percepção empírica que emerge de uma análise semiótica detalhada .

Conforme O'Halloran et al (2012), a análise de multimodalidade pode alcançar um grau de satisfação aceitável apenas se encaminhada a partir do uso de mídias interativas digitais e de técnicas computacionais que possam capturar de forma dinâmica os múltiplos desdobramentos semióticos combinados em diferentes modalidades de comunicação. Para os autores, o potencial de observação das ferramentas de análise multimodal e das metodologias de investigação associadas relaciona-se com o fato de que tais fenômenos codificam relações de natureza temporal e espacial.

Segundo Baldry e Thibault (2006a) e O’Halloran et al (2012), apesar de ter havido avanços importantes no desenvolvimento de técnicas de análise na área de linguística de corpora nos últimos anos, os aspectos de investigação de conteúdos multimodais são invariavelmente adaptados a partir de procedimentos analíticos e de orientação teórica voltados à observação de aspectos semióticos isolados. Em outras palavras, para os autores, essas abordagens de investigação são orientadas para a constituição de um olhar intra-semiótico frente à presença de mais de um canal de comunicação dentro de um corpus de análise, ao invés de abordar a coordenação de recursos semióticos próprios dessas interações. Além disso, este tipo de análise unidimensional deixa de olhar para outras interações que ocorrem para além do corpus, em nível intertextual ou transmidiático, como no caso de jogos digitais.

Existem inúmeros aparatos de codificação de corpora multimodais disponíveis, que podem variar desde o nível da segmentação de modalidade em um corpus, a esquemas de anotação exclusivos de apenas uma modalidade (escrita, imagética, sonora, etc.), descritivos de formas de comunicação como a linguagem gestual e a corporal, ou aplicadas a contextos situacionais, como a marcação de aspectos pragmáticos. Esses esquemas de marcação dizem respeito não só à estrutura de metadados utilizada, mas também à relação estabelecida entre diferentes aparatos teóricos e metodológicos usados para tornar mais eficiente a delimitação de recursos semióticos em um corpus multimodal.

Para Leech (2004), deve haver uma preocupação em se estabelecer uma padronização de categorização dos diferentes códigos de anotação utilizados na anotação de corpora. Tal afirmação não deve ser considerada apenas para corpora multimodais, mas para corpora de apenas uma modalidade. Essa padronização não se refere a uma uniformidade de rotulações dentro de um corpus apenas, mas antes a um conjunto de formatos que sejam reconhecidos por outros aplicativos de análise 
de corpora. Essa abordagem consensual de descrição e categorização de fenômenos, sejam linguísticos ou de outra natureza, não apenas se sustenta a partir de diretrizes de rigor de pesquisa e de alinhamento com outros aparatos teóricos e metodológicos, mas também tem o objetivo de favorecer a replicação e o compartilhamento dos resultados gerados durante o estudo.

Na maior parte das vezes a investigação de interações multimodais se dá a partir da observação de recursos semióticos isolados - via de regra, de imagens estáticas, comumente a partir da análise de fotogramas (frames) -, como forma de contornar a falta de ferramentas apropriadas ou o desconhecimento técnico para a análise de interações dinâmicas, como é o caso de games e arquivos em formato de vídeo. Segundo Baldry \& Thibault (2006, apud O’Halloran 2010)

[...] textos multimodais integram seleções de diferentes recursos semióticos a seus princípios de organização [...] Esses recursos não são simplesmente justapostos como modos distintos de significado, mas são combinados para constituir algo que em sua completude não pode ser reduzido ou explicado a partir da soma de suas partes distintas (p. 26, tradução minha ${ }^{1}$ ).

Para Kress e Van Leuuwen (2001, p. 20) o conceito de multimodalidade é pautado, a partir do uso de diferentes recursos semióticos, para a constituição de um evento ou de um produto. De maneira coordenada, esses recursos podem reforçar um ao outro, suprir de forma complementar funções semióticas ou estabelecer uma organização hierárquica entre diferentes aspectos de representação. Para a construção de um corpus multimodal, de forma semelhante à obtenção de um conjunto de dados para uma análise de um corpus de textos escritos, é necessário dar atenção a uma série de aspectos que delimitem medidas de obtenção dos corpora de análise, e os recursos de visualização e análise de dados gerados.

Hoje em dia há uma facilidade muito grande de obtenção de ferramentas que podem auxiliar o pesquisador a contornar as principais limitações no encaminhamento de pesquisas de conteúdos multimodais. Além das limitações associadas a metodologias e esquemas de anotações convencionadas e compartilháveis entre aparatos de marcação distintos, essas abordagens de pesquisa também encontram dificuldades de integração entre diferentes tipos de software de análise de corpora. Esse fato se dá a partir da presença de padrões de anotação distintos entre plataformas e níveis de investigação linguística, isto é, o que pode ser observado em um corpus através de uma ferramenta não necessariamente poderá ser observado a partir de outro aplicativo de análise. Por essa razão, é necessário que, ao fazer a escolha do aplicativo de investigação para a análise de corpora multimodais, o pesquisador considere a possibilidade de processamento do maior número de recursos semióticos analisáveis, evitando assim, por exemplo, a necessidade de se utilizar uma ferramenta para a análise de vídeo, outra para a análise de oralidade, dados em formatos de texto, e outros recursos semióticos que por ventura constituam o seu corpus (SCHMIDT, T.; ELENIUS, K. E TRILSBEEK, P., 2010).

De acordo com Salway (2010),

\footnotetext{
${ }^{1}$ multimodal texts integrate selections from different semiotic resources to their principles of organisation. [...] These resources are not simply juxtaposed as separate modes of meaning making but are combined and integrated to form a complex whole which cannot be reduced to, or explained in terms of the mere sum of its separate parts.
} 
Comparado com o sucesso obtido na análise de textos e na compreensão de linguagem, o uso de computadores para a análise automática de documentos multimodais estará limitado ao fato de que a imagen, o áudio e o vídeo são muito menos manejáveis em termos computacionais do que o texto (p. 52, tradução minha) $)^{2}$.

Para a investigação de corpora multimodais com um dinamismo de interações entre recursos semióticos maior, como é o caso de jogos digitais localizados, os quais podem apresentar variações em suas instâncias de interação de jogador para jogador, de uma partida para a outra, ou, especificamente, de uma versão original do jogo para a sua versão localizada, a tarefa de observação dos eventos que circunscrevem e descrevem diferentes recursos semióticos deve adotar uma perspectiva de observação que não seja estática e segmentada, mas igualmente dinâmica e coordenada, como já sugerido para corpora multimodais. Esse olhar aproximado para os múltiplos aspectos de representação que emergem em corpora multimodais requer desse tipo de investigação uma perspectiva de análise orientada à composicionalidade dos recursos semióticos visualizados e experienciados, entre a relação estabelecida entre o que é representacional e o que é interativo, e como esses elementos são integrados e se tornam relevantes para o espectador (ou, mais especificamente, para o jogador) (KRESS e VAN LEEUWEN, 1996).

Atualmente, os recursos de representação que fazem parte de interações em jogos digitais se manifestam de várias formas, sobretudo a partir de novos consoles. Estímulos táteis (a vibração de um joystick durante ações do jogador), a interação com o jogo em frente a uma câmera integrada ao console, a movimentação de um joystick em três dimensões como forma de estender os movimentos de um personagem ou um objeto no jogo são alguns exemplos de redimensionamentos de jogabilidade em jogos atuais. Em se tratando de investigações de jogos a partir de suas características multimodais, torna-se necessário o reposicionamento de aparatos teóricos e práticos na tarefa de descrever formas de representação que não são apenas visual, textual e sonora, mas que também incluem estímulos que vão além da tela (O’HAGAN E MANGIRON, 2013).

De acordo com O’Hagan e Mangiron (2013, p. 198), as diferentes formas de interação com o jogo citadas acima expandem de maneira significativa as dimensões de sua localização. Para as autoras, ainda que as exatas implicações dessas tecnologias para a localização de jogos não sejam claras, avanços dinâmicos nas tecnologias relacionadas a jogos inevitavelmente refletem em práticas de localização, abarcando questões de ordem linguística, tecnológica, cultural, e de dimensões sociais relacionadas à experiência de jogo.

Todos esses elementos unem-se para constituir a experiência do jogador, e, como um todo, idealmente, precisam ser transportados a um novo locale por meio da localização do game. Para criar uma experiência de jogo que seja equivalente à experiência do jogo original, a localização do game deve operar em todos os níveis, da manipulação linguística no nível micro até a do produto como um todo no nível macro, retendo não apenas a funcionalidade, mas também o apelo que intencionou-se ter o game ao usuário final (ibid, tradução minha) ${ }^{3}$.

\footnotetext{
${ }^{2}$ Compared with successes in text analysis and language understanding, the use of computers for the automated analysis of multimodal documents will be limited by the fact that image, video, and audio data are much less computationally tractable than text data.

${ }^{3}$ All of these elements come together to make up the gameplay experience of the player and, as a package, they ideally need to be transferred across to a new locale through game localization. In
} 
Para Dena (2010), a implementação dessa proposta de análise multimodal de jogos digitais ganha contornos ainda mais representativos, sobretudo pela característica estratificada, própria dessa mídia, e da natureza do conceito de localização assentado em paradigmas tradutórios e tecnológicos. Hoje, muitos jogos lançados caracterizam-se pela profundidade e dimensões fílmicas de seus enredos. Esse aspecto atribui ao estudo dessa mídia uma percepção narrativa expandida, que se estabelece não apenas dentro do jogo, mas que em muitos momentos faz-se a partir de características além de seu meio, de forma transmidiática, por meio de outras mídias que sustentam e dão continuidade ao jogo ou à sua história, como sugere Jenkins (2007). Para tanto, ao contemplar a análise dos vários aspectos multimodais e multimidiáticos circunscritos ao jogo, o pesquisador deve considerar como outras mídias (TV, filmes, HQs, livros, etc.) dão suporte e estendem a experiência do jogador e do próprio conteúdo de um jogo.

Diferentes níveis de análise podem ser contemplados no processo de investigação e anotação de corpora linguísticos. Todavia, é importante ressaltar que esses níveis de análise podem ser expandidos a partir da relação que se estabelece entre eles em um corpus multimodal. Di Giovani (2008) pontua que a percepção da imagem e da palavra inseridas em textos audiovisuais passa a adquirir um significado especial, por vezes indissociável, uma vez que se tornam, em termos tradutórios, veículos de representação cultural e identitária. O’Hagan e Mangiron (2013) chamam a atenção para o aspecto audiovisual de textos contemporâneos além de para como os diferentes recursos semióticos que os compõem se tornam relevantes culturalmente e favorecem uma perspectiva relevante para discussões sobre a localização de jogos e as relações de natureza tradutória a que estão circunscritos.

Por se tratar de uma perspectiva de investigação que sugere uma observação integral de todos os aspectos de interação e representação de sentidos envolvidos num jogo, e por sua natureza multimodal, a utilização de hardware e software específicos para a captura de dados de análise faz-se necessária. Para consoles de vídeo game como Xbox 360, PS3, Wii, Wii U, Xbox One, e PS4, essa tarefa demanda do pesquisador um investimento maior do que o da aquisição do próprio console. É necessária também a aquisição de uma placa de vídeo dedicada para o registro dos dados de análise em formato de vídeo, ou seja, da interação que ocorre durante o jogo, das falas dos personagens, de contextualizações narrativas em forma de textos, imagens, e várias outras instâncias de representação de significados que constituem essa mídia.

Placas de captura de vídeo internas (como a ilustrada na figura 1) podem funcionar a partir de um computador, integrada à sua placa-mãe e dependente de sua unidade de armazenamento interna (hard drive) ou de uma unidade de armazenamento externa (pendrive ou HD externo).

order to create a game experience that is equivalent to that provided by the original, game localization operates at all levels from linguistic manipulation at the micro level to the macro level of the product as a whole, retaining not only functionality, but the intended effective appeal to the end users (p. 198) 


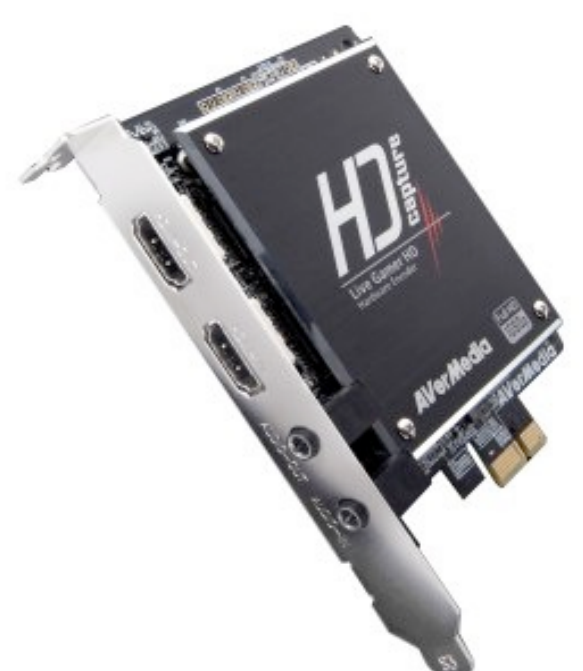

Figura 1 - Placa de captura interna (fonte ${ }^{4}$ )

Outra opção de equipamento para a captura de interações em jogos de consoles com o fim de análise de aspectos de multimodalidade pode ser feita a partir de uma placa de captura de vídeo externa. Este tipo de equipamento (como o ilustrado na figura 2) apresenta a grande vantagem de possuir uma unidade de processamento de imagem e captura dedicados, dando ao jogador/pesquisador uma maior segurança no registro de imagens e sons, uma vez que este não ficará dependente de um computador pessoal para o armazenamento de dados.

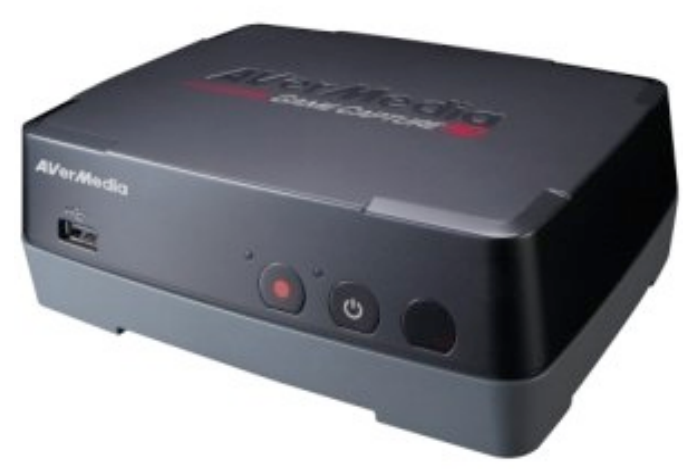

Figura 2 - Placa de captura externa (fonte ${ }^{5}$ )

Esses dois tipos de placa apresentam funcionalidades adicionais de um modelo para o outro, todavia, executam de maneira igual suas funções básicas de captura e armazenamento de dados em formato de arquivos de vídeo. Outras alternativas de menor custo para a captura de vídeo, como programas executados diretamente em um computador, podem ser utilizados para jogos de plataforma PC, Mac OS ou Linux ${ }^{6}$, como, por exemplo, Fraps ${ }^{\circledR}$, CamStudio ${ }^{\circledR}$, etc. Esses programas possuem as mesmas funcionalidades das placas de vídeo acima mencionadas,

4 http://www.avermedia.com/br/Product/ProductDetail.aspx?Id=467

5 http://avertv.avermedia.com/avertv/br/Product/ProductDetail.aspx?Id=470

$6 \mathrm{http} / /$ www.techgyd.com/top-10-free-screen-capturing-software/933/ 
todavia, muitos desses aplicativos podem apresentar algum atraso na coordenação entre áudio e vídeo, caso o usuário não possua um computador com um processamento satisfatório para rodar jogos mais atuais e com mais recursos gráficos.

\section{Ferramentas de anotação}

Abuczki e Ghazaleh (2013, p. 88) apontam que ferramentas de análise de corpora multimodais como ELAN, ANVIL e ExMERALDA oferecem uma série de benefícios para seus usuários uma vez que possibilitam a execução e a anotação de áudio, vídeo e/ou múltiplos arquivos de vídeo em janelas separadas, do mesmo programa, simultaneamente, a partir de esquemas de anotações convencionados e análise não convencionados. Ao definir qual plataforma de análise de dados utilizar, o pesquisador deve considerar a possibilidade de interoperabilidade de arquivos entre aplicativos de análises distintos. Em outras palavras, o pesquisador deve ter em mente que os dados gerados a partir de um programa de anotação podem ser utilizados para a replicação ou refinamento de resultados a partir de outras ferramentas de análise de multimodalidade, ou de ferramentas de análise de apenas uma modalidade. Essa preocupação perpassa não só o produto final de uma análise, mas as convenções de formatos de marcação adotadas, critérios acerca do tamanho do corpus de análise, sistema de transcrição, entre outros aspectos que possam assegurar o compartilhamento de conhecimento gerado para estudos posteriores (SCHMIDT, T.; ELENIUS, K. E TRILSBEEK, P., 2010).

O’Halloran (2009) sugere que novas tecnologias digitais podem representar novos paradigmas de investigação e visualização de recursos semióticos. Para Abuczki \& Esfandiari (2013, p.87), dois aspectos principais devem ser considerados ao se lidar com corpora multimodais: i) a natureza do processo de anotação de corpora multimodais disponíveis; ii) uma aparente deficiência nos aparatos e anotação existentes (ferramentas, formatos e esquemas) para a codificação de aspectos verbais e não-verbais.

Ainda, para Baldry e Thibauld (apud Hunston \& Thompson (2006)), o desenvolvimento de um aparato de anotação de corpora multimodais constitui-se em um desafio de ordem teória e, sobretudo, prática. Para os autores

Primeiramente, precisamos estabelecer os critérios teóricos para determinar os tipos de relações de significado pertinentes noutros sistemas semióticos muito menos estudados do que a linguagem, mas que frequentemente estão associados à ela num contexto de discurso particular para produzir os significados gerais que interpretamos. Em segundo lugar, não há solução automatizada direta ou fácil para a anotação de dados textuais. A transcrição e a anotação detalhada de textos multimodais é um processo que leva tempo e a análise automática não se mostra factível até o presente momento. Consequentemente, a coleta e a análise de dados é lenta (p.168, tradução minha). ${ }^{7}$

\footnotetext{
${ }^{7}$ First, we have to establish the theoretical criteria for determining the relevant kinds of meaning relations in other semiotic systems which are much less studied than language, but which often work together with language in particular discourse context to produce the overall meanings that we interpret. Secondly, there is no easy or straightforwardly automated solution to the question of tagging of the textual data. THe detailed multimodal transcription and tagging of multimodal texts is a time-consuming process and automatic parsing does not seem feasible at this early stage. Consequently, the collection and analysis of data is slow.
} 
Uma ferramenta muito útil para a anotação de interações multimodais, como é o caso de jogos digitais, é o aplicativo ELAN ${ }^{8}$. Originalmente utilizado para o estudo de línguas ameaçadas e língua de sinais, esse software permite a leitura e a anotação de corpora de forma estruturada e hierarquizada em diferentes níveis de marcação, chamados de trilhas (tiers) (fig. 3). Além da possibilidade de utilização de aparatos de anotação já convencionados, a ferramenta ELAN também permite a criação de sistemas de marcação personalizados, que atendam às necessidades e aos objetivos de pesquisa previamente estabelecidos, como é o caso da anotação de conteúdos localizados em jogos.

Dentre outras características desse programa que se destacam constam a tabulação de dados após a anotação; a flexibilidade de criação de estrutura de metadados; a organização hierárquica dos recursos semióticos observados em categorias e subcategorias; a anotação e a exportação de dados em formato XML; a interoperabilidade entre aplicativos semelhantes ou entre aplicativos de análise de apenas uma modalidade (por exemplo, Praat, entre outros programas que otimizam a tarefa de observação de textos dinâmicos como jogos) (SCHMIDT, T.; ELENIUS, K. E TRILSBEEK, P., 2010).

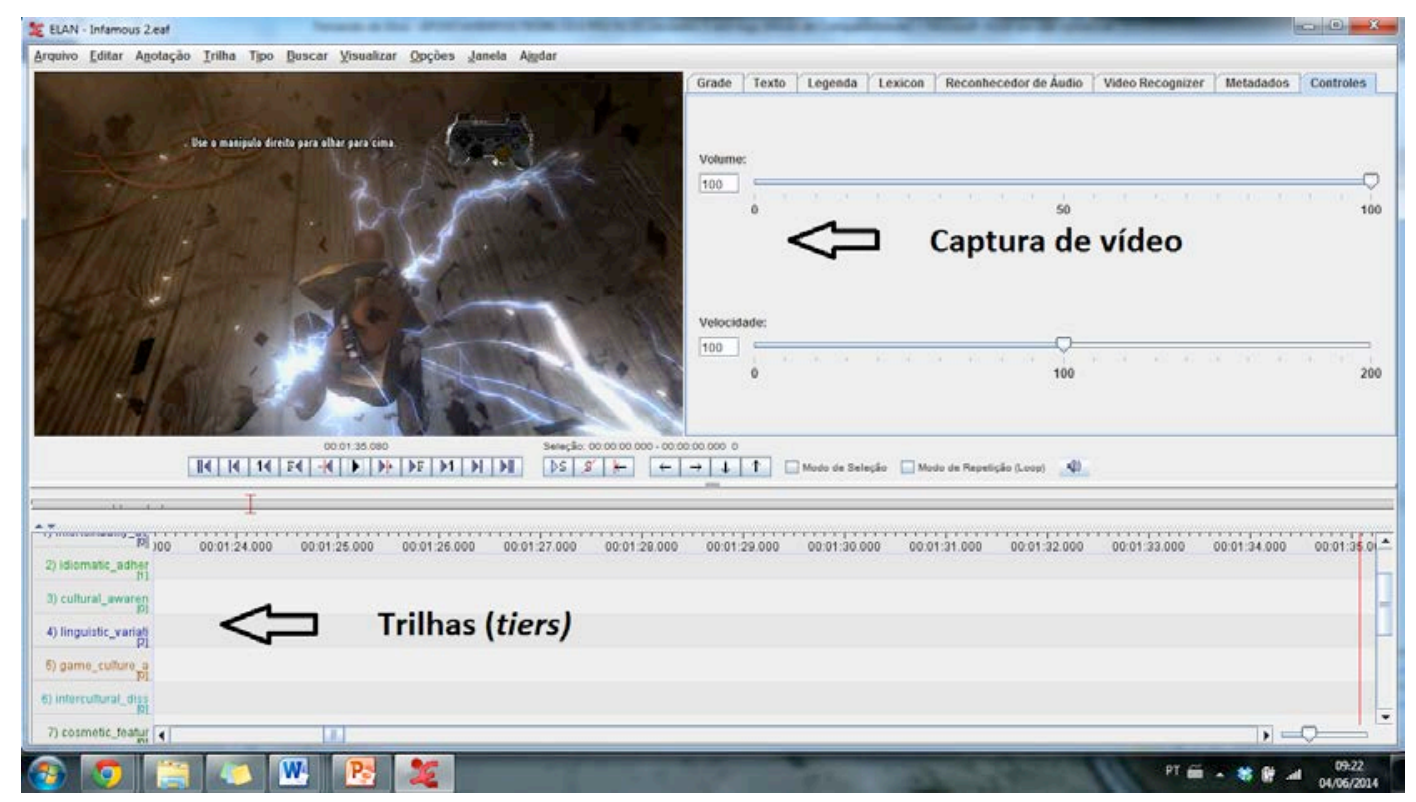

Figura 3 - ELAN

A possibilidade de lidar com diferentes tipos de corpora na mesma plataforma de análise, sem ter que dividir um corpus em diferentes segmentos para a investigação, torna-se útil no sentido de que evidencia as potencialidades do jogo para além de sua mídia e seu modo de expressão. Em outras palavras, um jogo pode, após seu lançamento, ganhar derivações de sua história e outras manifestações, que, se relevantes para a análise, podem ser referenciadas na mesma plataforma de investigação por meio de um aparato de marcação descritivo e relacional.

Nos últimos anos, linguistas tornaram-se cada vez mais conscientes do fato de que a investigação de corpora multimodais demanda cada vez mais a observação de seus diferentes domínios de comunicação - por exemplo, aspectos verbais e não verbais - como parte de um aparato descritivo de anotação multimodal. A anotação de interações multimodais ainda representa um desafio considerável uma vez que

8 http://tla.mpi.nl/tools/tla-tools/elan/ 
modos de comunicação distintos interagem mutuamente de forma dinâmica, e, muitas vezes, os limites entre um e outro podem não ser tão distinguíveis para o pesquisador (BLACHE; BERTRAND; FERRÉ, 2009).

Os parâmetros de investigação de interações multimodais - como jogos digitais -, por meio de metodologias de anotação de corpora não adquire um formato estanque, sobretudo pela ocorrência de simultaneidades entre seus diferentes modos de comunicação. A escolha acerca de qual ferramenta utilizar, bem como a de um esquema de anotação, dependerá de vários critérios e pode variar de acordo com as limitações do corpus anotado, dos recursos semióticos observados, bem como desde suas coocorrências à familiaridade do pesquisador com as metodologias de observação de corpora baseadas no uso das ferramentas citadas.

\section{Considerações finais}

Os elementos apontados neste texto, ainda que possibilitem lançar um olhar ampliado para os aspectos relacionados à tradução audiovisual, estudos de multimodalidade e estudos baseados em corpora, ainda não parecem receber o devido grau de reconhecimento na área dos Estudos da Tradução em seu sentido tradicional. Além dessa questão, para O’Halloran et al (2010a),

apesar do progresso que se tem feito no desenvolvimento da teoria e da descrição multimodal, assim como apesar da disponibilidade de poderosos recursos digitais cada vez mais sofisticados para o modelamento e a análise de fenômenos complexos, a maior parte das abordagens acerca da transcrição e análise de dados multimodais continuam a ser implementadas a partir de soluções com baixatecnologia, baseadas na observação de registros em página, metodologias que não apenas se mostram trabalhosas e demandam muito tempo do pesquisador, mas que limitam seriamente a habilidade de quem executa a tarefa de expor, descrever e investigar a complexidade da interação multifuncional de escolhas semióticas [...] (p. 10, tradução minha) ${ }^{9}$.

Para O’Halloran (2010) a análise de aspectos multimodais demanda o uso de técnicas que se caracterizem pela execução de uma investigação dos padrões integrativos de relações intersemióticas, que fazem parte do que a autora chama de “orquestração de escolhas semióticas em textos dinâmicos (p. 18)”. Para tanto, se torna necessária a utilização de tecnologias digitais que façam a análise coordenada de áudio, vídeo, texto e permitam ao mesmo tempo a anotação, mapeamento e a criação de um modelo estratificado desses múltiplos recursos de significação.

Como apontado neste texto, as ferramentas de análise disponíveis para a análise de multimodalidade, em termos práticos, ainda necessitam de um maior grau de automatização na execução de suas tarefas, além do fato de que os padrões de anotação, e o que pode ser anotado, ainda não estão por completo estabelecidos dentro de um aparato teórico e metodológico bem claro e definido, e os que já exis-

\footnotetext{
${ }^{9}$ Yet despite the progress that has been made in the development of multimodal theory and description, and the availability of increasingly sophisticated and powerful digital resources for modeling and analyzing complex phenomena, most approaches to the transcription an analysis of multimodal data continue to be implemented with 'low-tech', largely page-based, methodologies, which are not only extremely laborious and time-consuming to construct, but which severely constrain "the analysis ability to display, describe and analyze the complexity of the multimodal interplay of semiotic choices [...].
} 
tem, são derivativos de outras abordagens de análise. De acordo com Salway (2010) a menos que pesquisadores encontrem formas de estabelecer relações de associação e quantificação entre valores e recursos semióticos a partir de interfaces de análise multimodal, as abordagens empregadas a esse tipo de investigação serão, por muito tempo, relegadas ao nível de análises de fotogramas (frames) ou baseadas na inserção manual de metadados para posterior processamento por uma plataforma computacional.

\author{
Fernando da Silva ${ }^{10}$ \\ fe900571@gmail.com \\ Doutorando, Universidade Federal de Santa Catarina
}

\title{
Referências bibliográficas
}

ABuczKI, Ágnes; GHAZAleh, Esfandiari Baiat. An overview of multimodal corpora, annotation tools and schemes. Argumentum, Hungria, v. 1, n. 9, p.86-98, 2013.

BAldry, Anthony; ThiBault, Paul J.. Multimodal Transcription and Text Analysis: A multimedia toolkit and coursebook. Londres: Equinox, 2006a.

BAlDRY, Anthony; ThiBAult, Paul. Multimodal Corpus Linguistics. In: Hunston, Susan; ThOMPson, Charis (Ed.). System and Corpus: Exploring Connections. Birmingham: Equinox Publishing, 2006b. p. 164-182.

Blache, Philippe; Bertrand, Roxane; Ferré, Gaëlle. Creating and Exploiting Multimodal Annotated Corpora: The ToMA Project. In: KIPP, Michael et al (Org.) aqui falta a obra

DenA, Christy. Beyond Multimedia, Narrative, and Game: The controbutions of Multimodality and Polymorphic Fictions. In: PAGE, Ruth (Org.). New Perspectives on Narrative and Multimodality. Nova York: Routledge, 2010. p. 183-201.

Di Giovani, Elena. Translations, Transcreations and Transrepresentations of India in the Italian Media. Meta, Itália, v. 1, n. 53, p.26-43, 2008.

Jenkins, Henry. Transmedia Storytelling 101. 2007. Disponível em: $<$ http://henryjenkins.org/2007/03/transmedia_storytelling_101.html>. Acesso em: 03 jun. 2014.

\footnotetext{
${ }^{10}$ Fernando da Silva é bacharel em Letras de língua inglesa e literatura pela Universidade Federal de Santa Catarina (UFSC), mestre em Estudos de Tradução (PGET - UFSC) e doutorando pelo mesmo programa de pós-graduação. Atualmente faz sua pesquisa de doutorado com foco em jogos digitais localizados dentro do par linguístico inglês-português.
} 
Kress, Gunther; Van Leeuwen, Theo. Reaing Images: The Grammar of Visual Dsign. 2. ed. Londres e Nova York: Routledge, 1996. 321 p. . Multimodal Discourse: The Modes and Media of Contemporary Communication. Londres: Arnold Publishers, 2001

LeECH, G.. "Adding Linguistic Annotation" in Developing Linguistic Corpora: a Guide to Good Practice, 2004, ed. M. Wynne. Oxford: Oxbow Books: 1729. Disponível em: http://ahds.ac.uk/linguistic-corpora/

O'HAGAN, Minako; MangIron, Carmen. Game Localization. Amstedam/Filadélfia: John Benjamins, 2013. 386 p. (Volume 106).

O'HALlORAN, Kay et al. Challenges in designing digital interfaces for the study of multimodal phenomena. Information Design Journal, Holanda e Filadélfia, v. 18, n. 1, p.2-32, 2010.

. Interactive software for multimodal analysis. Visual Communication, Londres, v. 11, n. 3, p.363-381, ago. 2012. Disponível em: $<$ http://vcj.sagepub.com/content/11/3/363>. Acesso em: 20 fev. 2014.

. Challenges in designing digital interfaces for the study of multimodal phenomena. Information Design Journal, Austrália, 01 abr. 2010. p. 2-21. Disponível em: <http://www.ingentaconnect.com>. Acesso em: 21 jul. 2013.

SAlWAY, Andrew . The computer-based analysis of narrative and multimodality. In: PAGE, Ruth (Org.). New Perspectives on Narrative and Multimodality. Nova York: Routledge, 2010. p. 50-64. Disponível em: <http://goo.gl/UXIDXC>. Acesso em: 20 fev. 2014.

SChMidt, T.; EleniUs, K. \& TRILSBEEK, P. Multimedia Corpora (Media encoding and annotation). Draft submitted to CLARIN WG 5.7. as input to CLARIN deliverable D5.C-3 "Interoperability and Standards"

[http://www.clarin.eu/system/files/clarin-deliverable-D5C3_v1_5-

finaldraft.pdf] CLARIN, 2010. 